\title{
Effect of panretinal photocoagulation on serum levels of laminin in patients with diabetes: a prospective study
}

\author{
L Luis Masmiquel, Rosa Burgos, Carlos Mateo, Ramón Martí, Rosa M Segura, \\ Rafael Simó
}

\begin{abstract}
Backgroundlaim-Laminin, a major specific non-collagenous glycoprotein of basement membrane, has been proposed as an index of diabetic retinopathy and high serum concentrations have been reported in patients with proliferative diabetic retinopathy. On the other hand, panretinal photocoagulation (PRP) prevents the progression of severe diabetic retinopathy and reverses preretinal neovascularisation. The aim of the study was to investigate the effect of PRP on serum levels of laminin in patients with diabetes. Methods-20 patients with diabetes undergoing PRP and 15 patients with mild or moderate non-proliferative diabetic retinopathy in whom a PRP was not performed were included in the study. Serum laminin-P1 (Lam-P1), the largest pepsin resistant fragment of laminin, was determined by radioimmunoassay in each patient before starting PRP and 3 months after it was accomplished. Similarly, a baseline and a 4 month sample were analysed in the non-photocoagulated controls.
\end{abstract}

Results-Serum Lam-P1 concentrations obtained 3 months after PRP were significantly lower when compared with the ini-

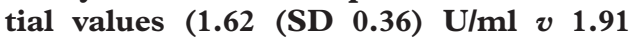
(0.37) U/ml; p <0.001). A decrease of serum levels of Lam-P1 could be seen in all patients. By contrast, in those patients with mild or moderate non-proliferative diabetic retinopathy in whom a PRP was not performed, no significant changes were detected in serum Lam-P1 concentrations (1.72 (0.20) U/ml $v 1.74(0.17)$;

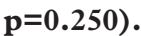

Conclusion-PRP decreases serum Lam-P1 levels in patients with severe diabetic retinopathy. Thus, the studies addressed to evaluate the usefulness of Lam-P1 as a marker of diabetic retinopathy should consider previous PRP as an influencing factor. Finally, our results suggest that retinal source of Lam-P1 strongly contributes to serum Lam-P1 in patients with severe diabetic retinopathy. (Br F Ophthalmol 1999;83:1056-1059)

Dr Rafael Simó, Diabetes

Unit, Endocrinology

Division, Hospital General

Universitari, Vall d'Hebron,

Vall d'Hebron 119-129,

08035 Barcelona, Spain.

Accepted for publication 4 May 1999
Diabetic microangiopathy is associated important disturbances in the metabolism of basement membranes. ${ }^{1}$ Because the serum levels of basement membrane (BM) constitu- ents could reflect these metabolic changes, their usefulness as markers of diabetic microangiopathy has been extensively investigated. In this regard, serum levels of laminin, a major specific non-collagenous glycoprotein of BM, have been proposed as an index of diabetic microangiopathy, ${ }^{2}$ and high concentrations have been reported in patients with diabetes with proliferative diabetic retinopathy. ${ }^{2}$ Notably, laminin contributes to the net increase in BMs that occurs during diabetic retinopathy (DR) and is one of the main constituents of the periodic acid Schiff positive material deposited in BMs of retinal vessels of patients with DR. ${ }^{4}$

Proliferative DR is characterised by the presence of complex mechanisms that favour degradation and synthesis of BM de novo. Proliferating endothelial cells digest BMs mainly proteolytically during the migratory phase of their growth and lay down new BM components as new vessels become established. ${ }^{56}$ Retinal ischaemia has a crucial role as a trigger of these phenomena. ${ }^{78}$ Panretinal photocoagulation (PRP) eliminates ischaemic areas and allows redistribution of blood flow to spared areas, thus improving the situation of retinal hypoxia and clinical studies have demonstrated the efficacy of PRP in preventing the progression of severe DR and reverting to preretinal neovascularisation..$^{8-10}$ Thus, it is tempting to speculate that PRP could decrease laminin serum concentrations in patients with diabetes with proliferative DR. However, PRP has not been previously considered as a variable that could influence serum laminin concentrations in patients with diabetic retinopathy (DR), and there are no studies focused on the effect of PRP on serum levels of laminin. Furthermore, because of the isolated intervention of laser treatment on ocular vessels, the effect of PRP on serum concentrations of laminin could provide a new insight into the contribution of the retinal source of laminin to its serum levels in patients with severe DR. On this basis, we have designed a prospective study to determine the effect of PRP on serum laminin-P1 (the largest pepsin resistant fragment of laminin) levels in patients with severe DR.

Patients and methods

Twenty consecutive patients undergoing bilateral PRP because of severe DR ( $>51$ according to the Wisconsin grading system ${ }^{11}$ ) were included in the study. Fifteen patients with mild or moderate non-proliferative DR and comparable for variables shown in Table 1 
served as a control group. Subjects presenting with additional diseases, ketosis or ketoacidosis in the past 3 months, uncontrolled blood pressure, or renal failure were excluded. The study was approved by the local ethics committee and all patients were fully informed before giving their consent.

Ophthalmological procedures were carried out by an experienced ophthalmologist. DR was evaluated by ophthalmoscopy and seven field colour retinographies with or without fluorescein angiography, and graded according to the method described by Klein et al. ${ }^{11} \mathrm{~A}$ clinical delivery system of the argon green laser (Aesculap Meditec CLKV 40E, Germany), with panfunduscopic and/or Goldmann lens when appropriate, was used for PRP. Each eye received approximately 2000-3000 burns, of 100-500 $\mu \mathrm{m}$ diameter, and 0.2 seconds' duration. Treatment was staged over three to five sessions (one session per week). Serum laminin-P1 (Lam-P1) concentrations were determined in each patient before starting PRP and 3 months after it was accomplished. Similarly, baseline and 4 month samples were analysed in the non-photocoagulated controls. This sample schedule was used to ensure that the process of laser wound healing was accomplished. An additional serum sample was obtained in patients who presented with signs of DR activity (recent appearance of new vessels or more than one of the followingmultiple haemorrhages, multiple cotton wool spots, venous irregularities, widespread closure with leakage of dye on fluorescein angiography) during 12 months of follow up after PRP.

Serum Lam-P1 was determined by a double antibody radioimmunoassay (CIS, Bio International) according to the method described by Brocks et al. ${ }^{12}$ Lam-P1 was obtained from pepsin digests of human placenta. Monovalent

Table 1 Clinical characteristics of the diabetic patients included in the study

\begin{tabular}{|c|c|c|c|c|}
\hline & \multicolumn{2}{|c|}{$\begin{array}{l}\text { Patients undergoing PRP } \\
(n=20)\end{array}$} & \multicolumn{2}{|c|}{$\begin{array}{l}\text { Patients not undergoing PRP } \\
(n=15)\end{array}$} \\
\hline & $n(S D)$ & Percentage & $n(S D)$ & Percentage \\
\hline Age (years) & $55.91(12.29)$ & - & $56.26(17.65)$ & - \\
\hline $\operatorname{Sex}(M / F)$ & $8 / 12$ & $40 / 60$ & $6 / 9$ & $40 / 60$ \\
\hline Type of diabetes (I/II) & $7 / 13$ & $35 / 65$ & $5 / 10$ & $33.3 / 66.7$ \\
\hline Duration of diabetes (years) & $21.35(7.41)$ & - & $20.46(8.7)$ & - \\
\hline \multicolumn{5}{|l|}{ Level of retinopathy ${ }^{\star}$} \\
\hline 31 & - & - & 10 & 66.7 \\
\hline 41 & - & - & 5 & 33.3 \\
\hline 51 & 6 & 30 & - & - \\
\hline 65 & 9 & 45 & - & - \\
\hline 70 & 5 & 25 & - & - \\
\hline \multicolumn{5}{|l|}{ Diabetic nephropathy† } \\
\hline No nephropathy & 9 & 45 & 7 & 46.7 \\
\hline Incipient & 6 & 30 & 5 & 33.3 \\
\hline Overt nephropathy & 5 & 25 & 3 & 20 \\
\hline \multicolumn{5}{|l|}{ Macroangiopathy $\ddagger$} \\
\hline No macroangiopathy & 11 & 55 & 9 & 60 \\
\hline Arterial occlusive disease & 7 & 35 & 5 & 33.3 \\
\hline Coronary heart disease & 5 & 25 & 3 & 20 \\
\hline
\end{tabular}

$\mathrm{PRP}=$ panretinal photocoagulation

*According to the Wisconsin grading system.

+Classified according to the mean urinary albumin excretion rate of three separate 24 hour urine collections: no nephropathy, $<20 \mu \mathrm{g} / \mathrm{min}$; incipient nephropathy, $20-200 \mu \mathrm{g} / \mathrm{min}$; overt nephropathy, $>200 \mu \mathrm{g} / \mathrm{min}$.

$\ddagger$ Diagnosis made on the basis of food pulse status, measurement of ankle pressure index, electrocardiogram, history of angor pectoris plus abnormal ergometry or previous myocardial infarction. Three patients with coronary heart disease (CHD) undergoing PRP and two patients with CHD not undergoing PRP also presented arterial occlusive disease.
Table 2 Serum Lam-P1 concentrations and haemoglobin $A_{1 c}\left(H b A_{1 c}\right)$ levels obtained at baseline and at the end of the study in diabetic patients in whom a PRP was performed (group $A)$ and in non-photocoagulated diabetic patients (group $B$ )

\begin{tabular}{llll}
\hline & Baseline & 3 months'follow up & p Value \\
\hline Group A & Lam-P1 & Lam-P1 & \\
& $1.91(0.37) \mathrm{U} / \mathrm{ml}$ & $1.62(0.36) \mathrm{U} / \mathrm{ml}$ & $<0.001$ \\
& $\mathrm{HbA}_{1 \mathrm{c}}$ & $\mathrm{HbA}_{\mathrm{cc}}$ & $\mathrm{ns}$ \\
& $8.4(1.4) \mathrm{g} / 1$ & $8.5(1.6) \mathrm{g} / 1$ & \\
Group B & $\mathrm{Lam}^{\mathrm{P} 1}$ & $\mathrm{Lam}-\mathrm{P} 1$ & \\
& $1.72(0.20) \mathrm{U} / \mathrm{ml}$ & $1.74(0.17)$ & $\mathrm{ns}$ \\
& $\mathrm{HbA}_{1 \mathrm{c}}$ & $\mathrm{HbA}_{1 \mathrm{c}}$ & $\mathrm{ns}$ \\
& $8.2(1.3) \mathrm{g} / 1$ & $8.1(1.2) \mathrm{g} / 1$ & \\
\hline
\end{tabular}

IgG fragments (Fab) obtained from rabbit antisera against Lam-P1 and goat antiserum to rabbit IgG were used as first and second antibodies, respectively. This assay is specific for laminin and no cross reactions were detectable with several collagens or fibronectin. Standards were analysed in triplicate and samples in duplicate. Our intra-assay and interassay coefficients of variation were $3.6 \%$ and $5.8 \%$, respectively. Statistical analysis was carried out using two tailed Student's test for paired data and the level of significance was set at $5 \%$. Data are expressed as mean (SD).

\section{Results}

The results are summarised in Table 2. Serum Lam-P1 concentrations obtained 3 months after PRP in patients with severe DR (group A) were significatively lower when compared with the initial values $(1.62(0.36) \mathrm{U} / \mathrm{ml} v 1.91$ (0.37) $\mathrm{U} / \mathrm{ml} ; \mathrm{p}<0.001)$; the decrease in serum Lam-P1 levels could be seen in all these patients. By contrast, in those patients with mild or moderate non-proliferative diabetic retinopathy (group B) in whom a PRP was not performed, no significative changes were detected in serum Lam-P1 concentrations after a 4 month period $(1.72(0.20) \mathrm{U} / \mathrm{ml} v 1.74$ $(0.17) ; p=0.250)$. In both groups of patients the metabolic control remained stable during the follow up.

During 12 months of follow up after PRP, two patients shown signs of diabetic retinopathy activity and in both cases an increase in serum Lam-P1 were detected (1.61 $v 1.76$ $\mathrm{U} / \mathrm{ml}$ and $1.52 v 1.73 \mathrm{U} / \mathrm{ml})$.

\section{Discussion}

The thickening of basement membrane in capillaries and small vessels is considered to be a characteristic histological finding of diabetic retinopathy. ${ }^{1}$ Laminin, unlike other adhesive proteins, is specific to BMs and contributes to the $\mathrm{BM}$ thickening. ${ }^{1}$ Because serum breakdown products of laminin are indicative of the turnover of basement membranes, the usefulness of serum Lam-P1 as a marker of diabetic retinopathy has been reported. Several pathogenic mechanisms could be implicated in the increase in serum Lam-P1 concentrations observed in patients with diabetic retinopathy. Firstly, there could be an enhanced synthesis of laminin; thus, increased amounts of laminin have been observed in retinal vessels of patients with diabetic retinopathy and increased mRNA for laminin has been demonstrated in endothe- 
lial cells and tissues exposed to high glucose concentrations. ${ }^{413-16}$ Secondly, there could be an increased enzymatic degradation of laminin; thus, DR is characterised by accelerated endothelial cell death which could favour the contact between $\mathrm{BM}$ and plasma proteases and promote the capillary occlusion phenomena. ${ }^{17}$ The inflammatory cells observed in great numbers in these occluded capillaries and microaneurysms can synthesise and activate enzymes with a capacity to degrade the basement membrane. ${ }^{18-20}$ Finally, and probably more importantly, BM catabolism is an obligatory step for retinal neovascularisation and, in this process, proliferating cells synthesise BMs with abnormal function and structure. ${ }^{21-23}$ Furthermore, in vitro models of neovascularisation suggest that neovessels show abnormal polarity and secrete part of $\mathrm{BM}$ components to the lumen. ${ }^{24}$

The effect of PRP on serum levels of Lam-P1 has not been investigated previously. In the present study we found a significant decrease in serum levels of Lam-P1 in patients with severe DR undergoing PRP. By contrast, no differences were observed in serum Lam-P1 concentrations in those non-PRP patients with mild or moderate non-proliferative $\mathrm{DR}$ that remained stable during follow up. Therefore, our results prove for the first time that PRP affects the serum concentration of a BM specific constituent (that is, laminin) in patients with diabetes. Certainly, this finding is intriguing when considering that retinal microcirculation represents only a minor part of the total number of blood vessels in the body. However, we previously demonstrated by multiple regression analysis, in a large diabetic population, that the most significant variable independently associated with serum Lam-P1 was diabetic retinopathy. ${ }^{3}$ In addition, some clinical evidence supports the idea that the retinal changes which occur after PRP in patients with proliferative diabetic retinopathy can modify significantly the circulating levels of some molecules. Jennings et $a l,{ }^{25}$ when exploring the oxidative status in the peripheral blood of patients undergoing PRP, demonstrated an acute increase in malondialdehyde-like material, plasma thiols, and red cell reduced glutathione. On the other hand, Hyer et al ${ }^{26}$ reported a decrease of serum IGF-1 concentrations in patients with proliferative diabetic retinopathy after effective PRP. Recently, Nishimura et al, ${ }^{27}$ observed in a cross sectional study that the mean serum concentration of human hepatocyte growth factor in diabetic patients with proliferative diabetic retinopathy, in whom a PRP was performed, was lower than in a proliferative diabetic retinopathy group without a history of photocoagulation. In the present study, Lam-P1 serum concentration decreased significantly after PRP to similar levels as those obtained in patients with non-proliferative diabetic retinopathy. Furthermore, it must be emphasised that we found a decrease in serum levels of Lam-P1 in all patients with diabetes undergoing PRP. Taken together, these results support the hypothesis that PRP could be useful in investigating the contribution of the retina to the circulating levels of several molecules in patients with proliferative diabetic retinopathy.

We can only speculate about the mechanisms involved in the the decrease of serum Lam-P1 observed in lasered patients. PRP eliminates important ischaemic areas and favours the redistribution of blood flow to uninjured ones, therefore decreasing retinal hypoxia, a crucial event in the aetiopathogenic cascade of diabetic retinopathy. Thus, PRP would reduce the angiogenic growth factor output from an ischaemic retina and therefore inhibit neovascularisation. ${ }^{89}$ In this way, several studies have demonstrated the efficacy of PRP in reversing retinal neovascularisation. ${ }^{8-10}$ Therefore, it seems possible that PRP reduces laminin serum levels by virtue of its neovascularisation reducing effect. Laminin secreted by proliferating cells could upregulate cell proliferation $^{28}$ and it has recently been shown that the laminin $67 \mathrm{LR}$ receptor is expressed preferentially by preretinal new vessels. ${ }^{29}$

Interestingly, our data suggest that the fluctuation of serum Lam-P1 levels in lasered patients could be related to DR activity. Thus, serum Lam-P1 concentration increased in those patients who developed signs of DR activity after the initial laser treatment. However, the small number of patients with DR activity post-PRP evaluated in this study does not allow us to be certain of the usefulness of serum Lam-P1 as an index of DR reactivation in photocoagulated patients, and further studies are needed.

1 Williamson JR, Tilton RG, Ghang K, et al. Basement membrane abnormalities in diabetes mellitus: relationship to clinical microangiopathy. Diabetes Metab Rev 1988;4:33970

2 Werle E, Diehl E, Hasslacher C. Levels and molecular size distribution of serum laminin in adult type I diabetic patients with and without microangiopathy. Metabolism 1998;47:63-9.

3 Simó R, Masmiquel LL, García Pascual L, et al. Serum concentrations of laminin-P1 in diabetes mellitus: usefulness as an index of diabetic microangiopathy. Diabetes Res Clin Pract 1996;32:45-53.

4 Bek T, Ledet T. Glycoprotein deposition in vascular walls of diabetic retinopathy. A histopathological and immunohistochemical study. Acta Opthalmol Scand 1996;74:38590 .

5 Vernon RB, Sage H. Between molecules and morphology. Extracellular matrix and creation of vascular form. $A m \mathfrak{F}$ Pathol 1995;147:873-83.

6 Pepper MS, Montesano R, Mandriota J, et al. Angiogenesis: a paradigm for balanced extracellular proteolysis during cell migration and morphogenesis. Enzyme Protein 1996; 49:138-62.

7 Kohner EM, Patel V, Rassam SMB. Role of blood flow and impaired autoregulation in the pathogenesis of diabetic retinopathy. Diabetes 1995;44:603-7.

8 Aiello LIP, Gardner TW, King GL, et al. Diabetic retinopathy. Diabetes Care 1998;21:143-56.

9 Grunwald JE, Brucker AJ, Petrig BL, et al. Retinal blood flow regulation and the clinical response to panretinal phoflow regulation and the clinical response to panretinal photocoagulation in proliferative
mology $1989 ; 96: 1518-22$.

10 Early Treatment Diabetic Retinopathy Study Research Group. Early photocoagulation for diabetic retinopathy. ETDRS report 9. Ophthalmology 1991;98(Suppl 5):76685.

11 Klein R, Klein BEK, Magli YL, et al. An alternative method of grading diabetic retinopathy. Ophthalmology 1986;93: 1183-7.

12 Brocks DG, Strecker H, Neubauer HP, et al. Radioimmunoassay of laminin in serum and its application to cancer patients. Clin Chem 1986;32:787-91.

13 Khono T, Sorgente N, Goodnight R, et al. Alterations in the distribution of fibronectin and laminin in the diabetic human eye. Invest Ophthalmol Vis Sci 1987;28:515-21.

14 Casaroli RP, Preissner K, Vilaró S. Fibronectin, laminin, vitronectin and their receptors at newly formed capillaries in proliferative diabetic retinopathy. Exp Eye Res 1995;60:5- 
15 Cagliero E, Roth T, Roy S, et al. Characteristics and mechanism of high-glucose-induced overexpression of basement membrane components in cultured human endothelial cells. Diabetes 1991;40:102-10.

16 Pugliese G, Pricci F, Pugliese F, et al. Mechanisms of glucose-enhanced extracellular matrix accumulation in rat glomerular mesangial cells. Diabetes 1994;43:478-90.

17 Mizutani M, Kern TS, Lorenzi M. Accelerated death of retinal microvascular cells in human and experimental diabetic retinopathy. $\mathcal{F}$ Clin Invest 1996;97:2883-90.

18 Engerman RL. Pathogenesis of diabetic retinopathy. Diabetes 1989;38:1203-6.

19 Ley K. Molecular mechanisms of leucocyte rolling and adhesion to microvascular endothelium Eur Heart $f$ 1993;14(Suppl 1):68-73.

20 Stitt AW, Gardiner TA, Archer DB. Histological and ultrastructural investigation of retinal micr ultrastructural investigation of retinal microaneurysm develop

21 Khono T, Sorgente N, Goodnight R, et al. Alterations in the distribution of fibronectin and laminin in the diabetic distribution of fibronectin and laminin in the diabetic
human eye. Invest Ophthalmol Vis Sci 1987;28:515-21.

22 Mandarino LJ, Sundarraj N, Finlayson J, et al. Regulation of fibronectin and laminin synthesis by retinal capillary endothelial cells and pericytes in vitro. Exp Eye Res 1993;57:609-21.
23 Casaroli RP, Preissner K, Vilaró S. Fibronectin, laminin, vitronectin and their receptors at newly formed capillaries in proliferative diabetic retinopathy. Exp Eye Res 1995;60:517

24 Madri JA, Pratt BM. Endothelial cell-matrix interactions : in vitro models of angiogenesis. $\mathcal{F}$ Histochem Cytochem 1986;34:85-91

25 Jennings PE, McEwen CJ, Fallon TJ, et al. Oxidative effects of laser photocoagulation. Free Radic Biol Med 1991;11: 727-30.

26 Hyer SL, Sharp PS, Brooks RA, et al. A two year follow up study of serum insulin like growth factors in diabetics with retinopathy. Metabolism 1989;38:586-9.

27 Nishimura $M$, Nakano $K$, Ushiyama $M$, et al. Increased serum concentrations of human hepatocyte growth factor in proliferative diabetic retinopathy. 7 Clin Endocrinol Metab 1998;83:195-8.

28 Kalfa TA, Gerritsen ME, Carlson EC, et al. Altered proliferation of retinal microvascular cells on glycated matrix. Invest Ophthalmol Vis Sci 1995;36: 2358-67.

29 Stitt AW, McKenna D, Simpson DA, et al. The 67-kd laminin receptor is preferentially expressed in proliferating retinal vessels in a murine model of ischemic retinopathy. Am F Pathol 1998;152: 1359-65. 